\title{
Stereoselective analysis of drugs and metabolites by the Chromatographic and Electrophoretic Analysis Center group
}

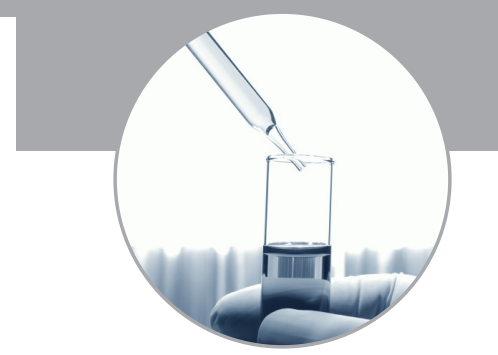

The Chromatographic and Electrophoretic Analysis Center group is based in the Faculty of Pharmaceutical Sciences of Ribeirão Preto, University of São Paulo, and is led by Pierina Sueli Bonato. Over the last two decades, the group has contributed in the area of enantioseparations by using modern analytical and sample preparation techniques for the analysis of chiral drugs and metabolites in biological matrices. In addition, the group has also been involved in the evaluation of stereoselective metabolism of drugs by using in vitro and biomimetic models.

Pierina Sueli Bonato joined the Faculty of Pharmaceutical Sciences of Ribeirão Preto, University of São Paulo, in 1980 and currently serves as a full-time professor in the Department of Physics and Chemistry. Her first research area of interest was centered on the use of chromatographic techniques for the analyses of drugs and metabolites in biological samples with application to analytical toxicology. In 1990, through collaborative projects with Piero Salvadore (Department of Chemistry and Industrial Chemistry, University of Pisa), Carlo Bertucci (Department of Pharmaceutical Sciences, University of Bologna) and Vera Lucia Lanchote (Faculty of Pharmaceutical Sciences of Ribeirão Preto, University of São Paulo), she started working in the exciting area of enantioseparations. Over the last two decades, her group, known as the Chromatographic and Electrophoretic Analysis Center, has contributed to this area by employing modern analytical and sample preparation techniques for the analysis of chiral drugs and metabolites in biological samples. In addition, this group has also been involved in studying stereoselective metabolism of drugs by using in vitro and biomimetic models.

\section{Modern techniques for the steroselective analysis of drugs \& metabolites}

The impact of stereochemistry in the design, discovery, development, launch and marketing of new drugs is highly recognized. Due to the stereoselective differences in the pharmacokinetic and pharmacodynamic properties of enantiomers and the emergence of new technologies that allow the preparation of pure enantiomers in significant quantities, the number of new drugs marketed as pure enantiomers has increased. In addition, recently the chiral-switch process has also been explored. In this process, drugs that have already been claimed, approved and/or marketed as racemates or as mixtures of diastereomers are now redeveloped as single enantiomers. As a consequence, reliable methods for the stereoselective analysis of drugs and metabolites in biological samples are required to support the decision of producing the drug as a single enantiomer or replacing the racemate by the single isomer.

The main techniques used for chiral separation of stereoisomers are GC, supercritical fluid chromatography (SFC), CE and HPLC. Among them, HPLC is the most widely explored. The resolution of enantiomers by HPLC can be achieved by mainly using chiral stationary phases. Although, chiral stationary phases can be prepared by using a broad variety of natural and synthetic chiral compounds, the most important ones are those based on polysaccharide derivatives and macrocyclic antibiotics. These chiral stationary phases have been successfully used by the group for the development of methods for the analysis of acidic, basic or neutral drugs and metabolites. Almost 70 drugs and metabolites have been analyzed in plasma, urine and liver microsomal fractions, yielding fully validated methods (Figure I).

In order to improve the selectivity and detectability of the methods for the chiral analysis of drugs in biological materials, in recent years, the group has employed LC-MS. The hyphenation of LC and MS (LC-MS and LC-MS/ MS) has given an enormous contribution to the analysis of drugs in biological samples. By using this technique, methods with high detectability and selectivity could be developed for analytes with different characteristics. In addition, high-throughput methods are very common

\section{Pierina Sueli Bonato}

Faculty of Pharmaceutical

Sciences of Ribeirão Preto,

University of São Paulo,

Ribeirão Preto, 14040-903,

Brazil

Tel.: +55 I6 3602426 I

Fax: +55 I6 36024880

E-mail: psbonato@fcfrp.usp.br

Chiral STATIONARY PHASES

Material that discriminates between stereoisomers allowing their

chromatographic separation 


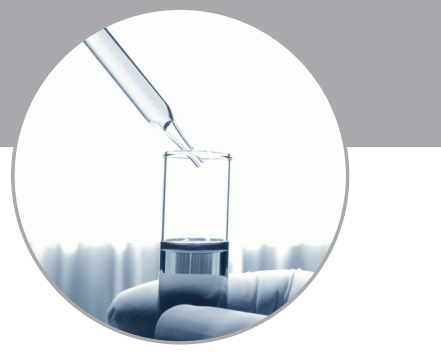

because the resolution of the parent drug and its metabolites is not necessary, since these analytes are usually distinguished by the detector. Due to this detector specificity, the interference of endogenous compounds is not a major problem, unless ionization suppression occurs. Additional and unique structure-related information regarding the intended drug or metabolite is also an important feature of this technique.

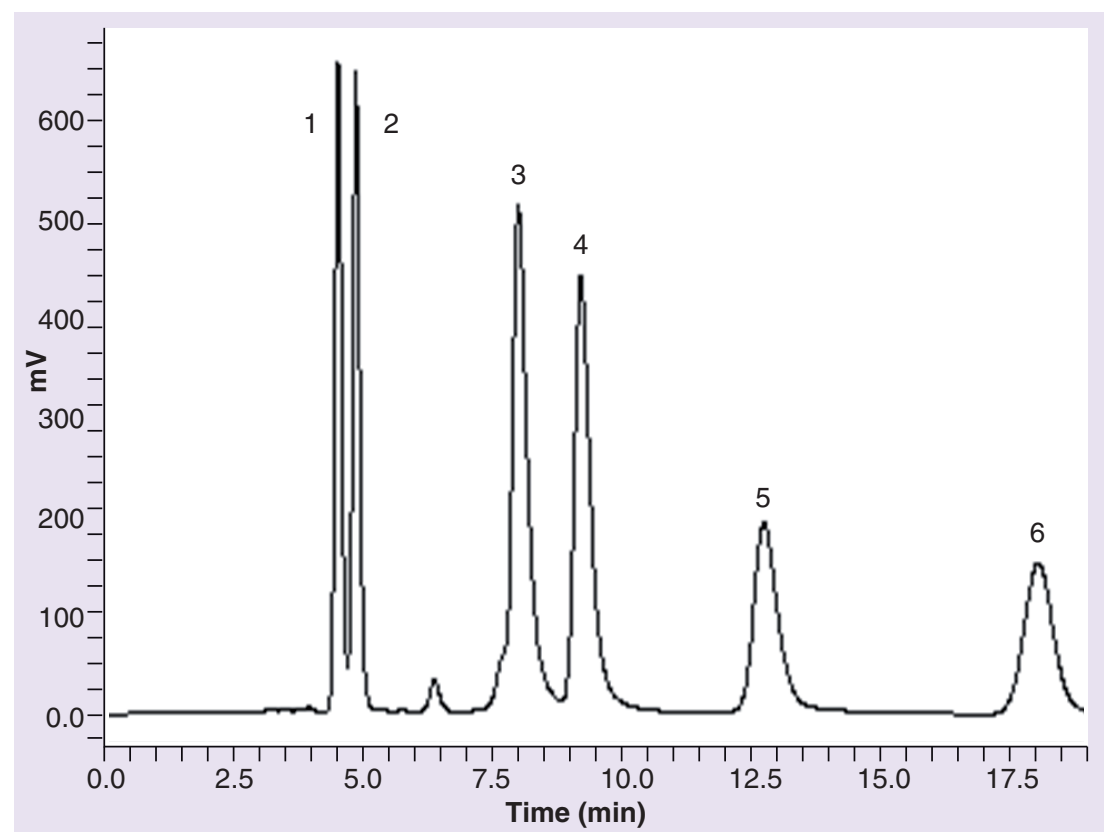

Figure 1. Chiral HPLC resolution of venlafaxine ((R)-VF [1] and (S)-VF [2]) and its metabolites $N$-desmethylvenlafaxine ( $R$ - -NDV [3] and (S)-NDV [4]) and O-desmethylvenlafaxine ((R)-ODV [5] and (S)-ODV [6]) on a Chiralpak ${ }^{\circledR}$ AD column.

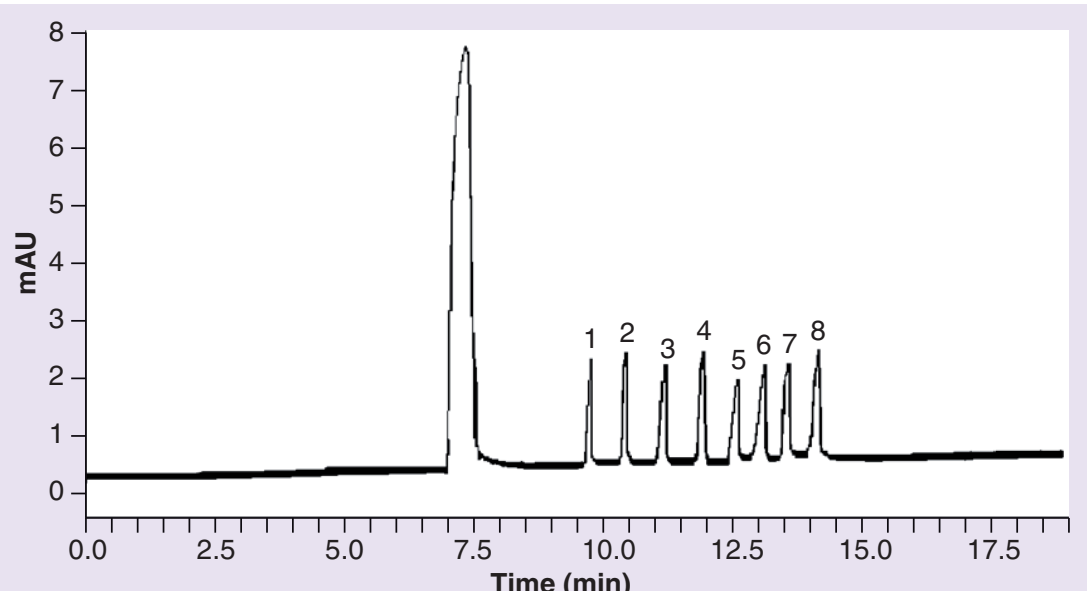

Figure 2. Capillary electrophoresis chiral resolution of hydroxycloroquine and its metabolites using hydroxypropyl- $\beta$-cyclodextrin and sulfated- $\beta$ cyclodextrin as chiral selectors. 1: $(R)$-hydroxychloroquine;

2: $(S)$-hydroxychloroquine; 3: $(R)$-desethylhydroxychloroquine;

4: (S)-desethylhydroxychloroquine; 5: $(R)$-desethylchloroquine;

6: (R)-bisdesethylchloroquine; 7: (S)-desethylchloroquine;

8: (S)-bisdesethylchloroquine.
Enantioselective analysis using LC-MS requires special care with the selection of the mobile phase. Highly aqueous mobile phases or the use of nonvolatile buffers or additives could cause ionization suppression or operational problems, whereas mobile phases based on hexane can be dangerous, especially for the atmospheric pressure chemical-ionization interface. To solve these problems, postcolumn addition of suitable solutions, such as ammonium acetate buffer/isopropanol, has been used.

Although HPLC with chiral stationary phases is a well-established technique for the analysis of chiral compounds in complex matrices, it presents two important drawbacks: the cost of the chiral columns and the use of high amounts of organic solvents. On the other hand, $\mathrm{CE}$ is a growing analytical technique that could be used for this purpose without these disadvantages, once the chiral separation is accomplished by using a chiral selector in the running buffer. CE requires only milliliter amounts of analysis solution, allowing the use of minimal amounts of expensive chiral selectors and resulting in a small amount of waste, mostly of aqueous buffer. In addition, other important features of CE, such as high separation efficiency, relatively easy method development, speed and simplicity of instrumentation, have made this technique very attractive for the analysis of chiral compounds in biological samples.

Several compounds have been used as chiral selectors in $\mathrm{CE}$, including cyclodextrins (CDs) and their derivatives, chiral crown ethers, macrocyclic antibiotics and chiral surfactants. Among these selectors, CDs and their derivatives are the most commonly used. The CDs are shaped like a truncated cone with a hydrophobic cavity and hydrophilic exterior due to the presence of hydroxyl groups in the opening of the truncated cone. The accepted mechanism of enantioresolution with CDs involves the inclusion of the chiral analyte in the cavity and the participation of secondary interactions with hydroxyl groups or other groups introduced by derivatization. These interactions differ between the stereoisomers, leading to a difference in their migration times and yielding their resolution in the analysis.

Due to the significant contribution of the Chromatographic and Electrophoretic Analysis Center group concerning the application of $\mathrm{CE}$ in the chiral analysis of drugs and metabolites in biological samples (Figure 2), Dr Bonato had the opportunity to be the guest editor of three special issues focused on enantioseparation in the Electrophoresis journal (2005, 2007 and 2009). 


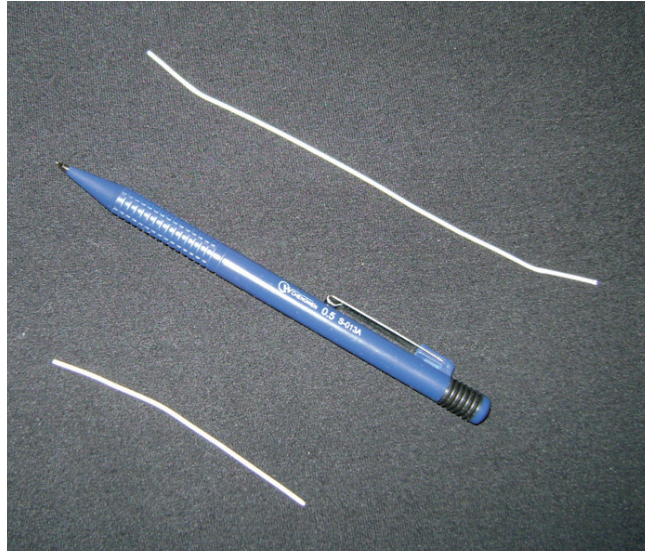

Figure 3. Hydrophobic membrane used in liquid-phase microextraction.

The determination of analytes present at trace levels in complex matrices usually requires previous processes for sample preparation. The techniques most commonly used for this purpose are liquid-liquid extraction and solid-phase extraction. However, there is an increased interest in using methods that are less harmful to the environment, generating less waste and employing minimum or no organic solvent. Within this context, microextraction techniques have been developed and one of the most attractive is hollow-fiber liquid-phase microextraction (LPME). In LPME, the pores of a hydrophobic capillary membrane (FIgURE 3) are impregnated with an organic solvent and its lumen is filled with an acceptor phase. The LPME may be accomplished in two or three phases. In the two-phase system, the analyte is extracted from the aqueous sample (donor phase) through an organic solvent immobilized in the pores of the membrane, to the same organic solvent (acceptor phase) present in its lumen. In the three-phase system, the acceptor phase is an aqueous solution, with $\mathrm{pH}$ opposite to the donor phase.

This technique has been extensively used by the Chromatographic and Electrophoretic Analysis Center group for the analysis of chiral drugs and metabolites in biological samples. The suitable recoveries obtained allowed the quantification limits necessary for the application of the methods in pharmacokinetic and metabolism studies to be reached (TABLE I).

\section{Method application in pilot pharmacokinetic studies \& in vitro or biomimetic metabolism evaluation}

The influence of stereochemistry in pharmacokinetics is remarkable, especially the metabolism step, since the interaction of the drug with enzymes is usually stereospecific. Drugmetabolism evaluation can be carried out by using in vivo models through the administration of therapeutic doses of the drugs to animals, healthy subjects or patients, followed by the assessment of plasma or urinary concentrations of the drug and its metabolites. Several methods developed by the Chromatographic and Electrophoretic Analysis Center group have been used in pilot pharmacokinetic studies in healthy volunteers or animals.

Table 1. Analytical performance of methods based on liquid-phase microextraction for the enantioselective analysis of drugs and metabolites in biological matrices.

\begin{tabular}{|c|c|c|c|c|c|c|}
\hline Drug/metabolite & Matrix & $\begin{array}{l}\text { Analytical } \\
\text { technique }\end{array}$ & LPME system & Recovery (\%) & $\begin{array}{l}\text { Quantification } \\
\text { limit (ng/ml) }\end{array}$ & Ref. \\
\hline Mirtazapine & Plasma & HPLC-UV & Two phase & 29 & 6.25 & [1] \\
\hline Hydroxycloroquine and metabolites & Urine & CE-UV & Three phase & $\begin{array}{l}86 \mathrm{HCQ} \\
84 \mathrm{DHCQ}, \mathrm{BDCQ} \\
92 \mathrm{DCQ}\end{array}$ & $10 ; 21$ & [2] \\
\hline Mirtazapine and metabolites & Urine & CE-UV & Three phase & $\begin{array}{l}24 \text { metabolites } \\
78 \text { mirtazapine }\end{array}$ & 62.5 & [3] \\
\hline Mirtazapine and metabolites & Plasma & LC-MS/MS & Three phase & $\begin{array}{l}30 \text { metabolites } \\
43 \text { mirtazapine }\end{array}$ & 1.25 & [4] \\
\hline Mefloquine & Plasma & HPLC-UV & Three phase & 34 & 50 & [5] \\
\hline Cloroquine and metabolites & Plasma & LC-MS/MS & Three phase & $\begin{array}{l}29 \mathrm{CQ} \\
48 \mathrm{DCQ} \\
66 \mathrm{BDCQ}\end{array}$ & 5 & [6] \\
\hline Oxybutynin and metabolite & Microssomal fraction & HPLC-UV & Three phase & $\begin{array}{l}60 \text { oxybutinin } \\
73 \text { metabolite }\end{array}$ & 250 & [7] \\
\hline Mefloquine and carboxymefloquine & Plasma & HPLC-UV & $\begin{array}{l}\text { Three phase } \\
\text { Two step }\end{array}$ & $\begin{array}{l}36 \text { mefloquine } \\
39 \text { metabolite }\end{array}$ & 50 & {$[8]$} \\
\hline
\end{tabular}



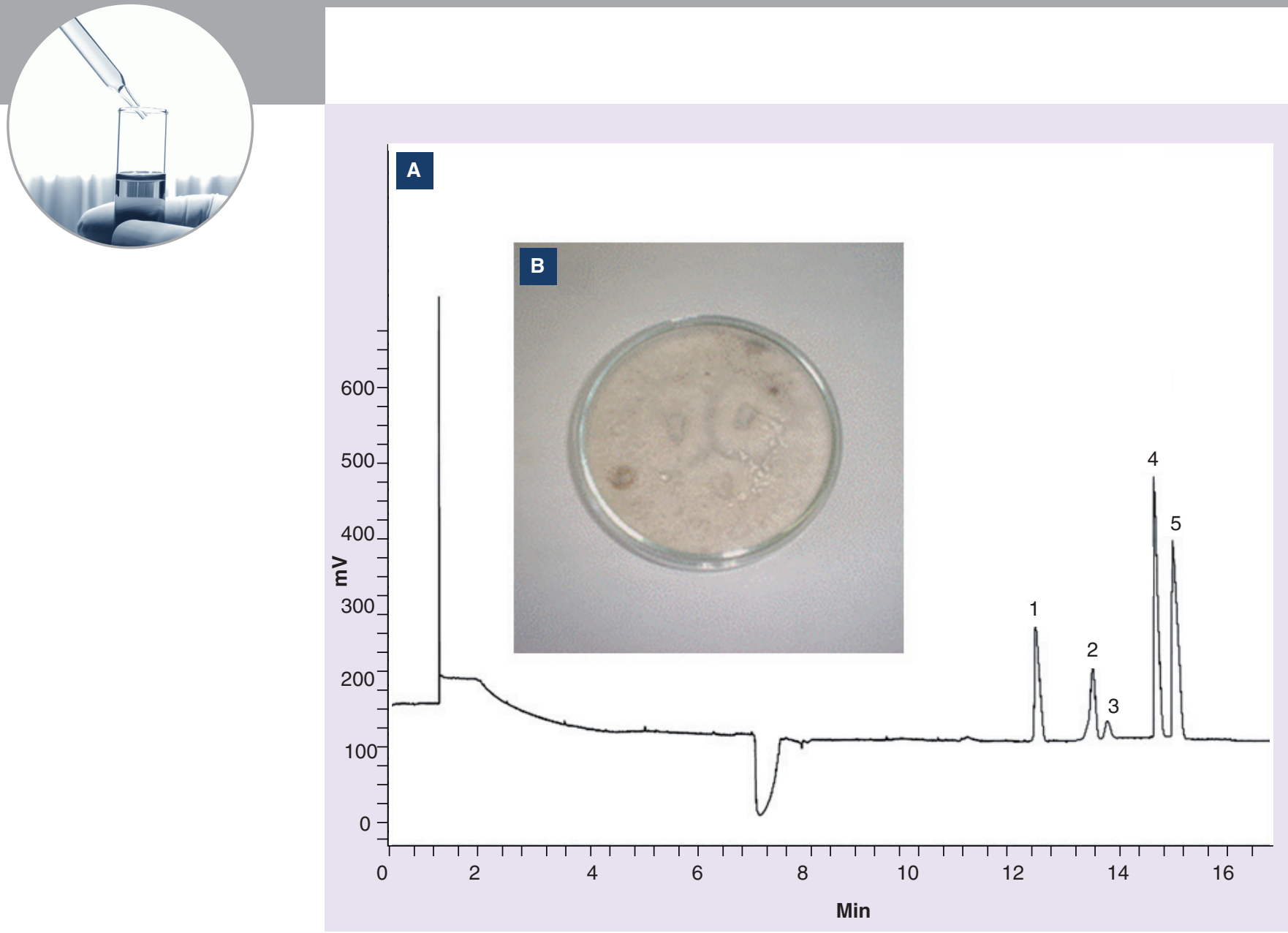

ENDOPHYTIC FUNGI

Fungi living inside the health plant tissues without causing any apparent disease

Figure 4. (A) Electropherogram showing the stereoselective biotransformation of propranolol by the endophytic fungus Phomopsis sp. (B) (1) Acet, (2) (S)-4-OH-Prop, (3) (R)-4-OH-Prop, (4) (S)-Prop and (5) (R)-Prop.

The metabolism mediated by cytochrome P450 can also be studied by using in vitro models. The development of in vitro models to study the metabolism was originated on ethical, scientific and economic aspects. In vitro methods are particularly useful for the rational selection of animal species for toxicological studies, to compare the metabolic profile and to evaluate the influence of different species in the metabolism. In addition, this model is also important for the production of metabolites for the development of bioanalytical methods. The group has evaluated the stereoselective metabolism of two antimalarial drugs (primaquine and hydroxychloroquine) and oxybutinin by using microsomal and mitochondrial preparations.

An alternative to in vitro metabolism studies is the use of biomimetic systems that mimic the action of cytochrome P450 enzymes. These systems are much simpler, allowing the biotransformation study to be held under controlled conditions and allowing the production of large quantities of metabolites.
Several years ago, the Chromatographic and Electrophoretic Analysis Center group employed metalloporphyrins to study the metabolism of certain drugs, such as praziquantel and disopyramide. More recently, the group is interested in studying the stereoselective biotransformation of drugs by using microorganisms, particularly endophytic fungi (fungi that live inside healthy plant tissues without causing any apparent disease). The most important advantages of using microorganisms in biotransformation processes are the relatively mild conditions of the procedures, environmental safety and, especially, high selectivity. Many mammalian Phase I and II metabolic reactions, including hydroxylation, $\mathrm{O}$ - and $\mathrm{N}$-dealkylation, dehydrogenation and glucuronide and sulfate conjugation, also occur in microbial models. Since 2005, the group has used endophytic fungi for the biotransformation of several drugs including thioridazine, propranolol (FIGURE 4), midrodrine and fluoxetine. We have observed that these microorganisms could metabolize the drugs producing the same, as well as different, mammalian metabolites. Generally, the metabolism is stereoselective. 


\section{Future perspective}

One of the main areas of focus in bioanalytical laboratories is the development of high-throughput methods by using automated systems for sample preparation, fast separation columns (short columns with small-diameter particles) and selective detection systems (LC-MS/MS or CE-MS/ MS). Considering the enantioselective analysis of drugs and metabolites, the use of LC-MS/ MS and CE-MS/MS for method development is expected to increase in the next few years. The development of miniaturized sample preparation procedures will also be focused on by the group in the future, allowing a considerable reduction in the use of organic solvents.

Particular attention will be given to the use of the developed methods to study the biomimetic biotransformation of drugs by using endophytic fungi. These microorganisms have shown great potential for the production of enantiomerically pure drug metabolites.
To develop the projects on these issues, the Chromatographic and Electrophoretic Analysis Center group intends to strengthen collaboration with the groups we are currently working with, as well as forming new collaborations with other groups.

\section{Financial \& competing interests disclosure}

The author would like to acknowledge financial support from Conselho Nacional de Desenvolvimento Cientifico e Tecnológico and Fundação de Amparo à Pesquisa do Estado de São Paulo. The author has no other relevant affiliations or financial involvement with any organization or entity with a financial interest in or financial conflict with the subject matter or materials discussed in the manuscript. This includes employment, consultancies, honoraria, stock ownership or options, expert testimony, grants or patents received or pending, or royalties.

No writing assistance was utilized in the production of this manuscript.

\section{Executive summary}

- The use of HPLC with chiral stationary phases, LC-MS, capillary electrophoresis and microextraction techniques for sample preparation are discussed.

- The application of the methods in pilot pharmacokinetic studies and in vitro or biomimetic metabolism evaluation is presented.

\section{Bibliography}

1 De Santana FJM, de Oliveira ARM, Bonato PS. Chiral liquid chromatographic determination of mirtazapine in human plasma using two-phase liquid-phase microextraction for sample preparation. Anal. Chim. Acta 549, 96-103 (2005).

2 de Oliveira ARM, Cardoso CD, Bonato PS. Stereoselective determination of hydroxychloroquine and its metabolites in human urine by liquid-phase microextraction and CE. Electrophoresis 28, 1081-1091 (2007).

3 de Santana FJM, Lanchote VL, Bonato PS. Capillary electrophoretic chiral determination of mirtazapine and its main metabolites in human urine after enzymatic hydrolysis. Electrophoresis 29, 3924-3932 (2008).

4 de Santana FJM, Bonato PS.

Enantioselective analysis of mirtazapine and its two major metabolites in human plasma by liquid chromatography-mass spectrometry after three-phase liquid-phase microextraction. Anal. Chim. Acta 606, 80-91 (2008).

5 Magalhães IRS, Bonato PS. Liquid-phase microextraction combined with highperformance liquid chromatography for the enantioselective analysis of mefloquine in plasma samples. J. Pharm. Biom. Anal. 46, 929-936 (2008).
6 Magalhães IRS, Bonato PS. Enantioselective determination of chloroquine and its $\mathrm{N}$-dealkylated metabolites in plasma using liquid-phase microextraction and LC-MS. J. Sep. Sci. 31, 3106-3116 (2008).

7 da Fonseca P, de Freitas LAP, Pinto LFR, Pestana CR, Bonato PS. Enantioselective analysis of oxybutynin and $N$-desethyloxybutynin with application to an in vitro biotransformation study. J. Chromatogr. $B$ 875, 161-167 (2008).

8 Magalhães IRS, Bonato PS. Two-step liquid-phase microextraction and highperformance liquid chromatography for the simultaneous analysis of the enantiomers of mefloquine and its main metabolite carboxymefloquine in plasma. Anal. Bioanal. Chem. 393, 1805-1813 (2009). 